\title{
Molecular Quantification of Pseudomonas Aeruginosa from Respiratory Samples in Patients with Suspected Ventilator Associated Pneumonia
}

\author{
Sahar Fayed $^{\mathrm{a}}$, Shahen Dabour ${ }^{\mathrm{b}}$, Heba Sallam $^{\mathrm{a}}$, Neveen Soliman ${ }^{\mathrm{a}}$
}

a

Department of clinical and chemical pathology, Faculty of Medicine, Benha University, Egypt. b Department of Paediatrics, Faculty of Medicine Benha University.

Correspondence to: Heba Sallam, department of clinical and chemical pathology, Faculty of Medicine, Benha university, Egypt.

Email:

ashrafgabr18@yahoo.com

Received: 22 March 2020

Accepted: 12 February 2021

Abstract:

Background: Ventilator-associated pneumonia (VAP) is a frequent issue in intensive care units (ICU), with a major impact on morbidity, mortality and cost of care. VAP diagnosis remains challenging: traditional culture-based microbiological techniques are still the gold-standard, but are too slow to enable clinicians to improve prognosis with timely antimicrobial therapy adjustment. Aim: to compare the performance of the real time quantitative molecular based method versus conventional culture in diagnosis of ventilator associated pneumonia caused by $\mathrm{P}$. aeruginosa in pediatric patients. Methods: this study included forty paediatric patients aged from one month to 18 years attending Paediatric Intensive Care Unit (PICU) and developed VAP during mechanical ventilation, All patients were subjected to full history taking, full clinical examination, chest X-ray, semiquantitative culture of BAL and ETA on different routine bacteriological media for isolation of the causative organisms and molecular quantification for detection and quantification of P. aeruginosa in BAL and ETA samples by real -time PCR. Results: The mean age in patients' group was $40.7 \pm 31.6$ months with male predominance $(60 \%)$. PCR ETA can diagnose Pseudomonas aeruginosa in VAP, with $88.9 \%$ sensitivity, $90.3 \%$ specificity, $72.7 \%$ PPV and 96.5\% NPV. AUC $(95 \% \mathrm{CI})=0.896(0.76-1.0)$ and ROC curve analysis showed that PCR BAL can diagnose Pseudomonas aeruginosa in VAP, with 100\% sensitivity, 96.7\% specificity, 90.9\% PPV and 100\% NPV. AUC $(95 \% \mathrm{CI})=0.983(0.94-1.0)$. Conclusion: Molecular biology makes it possible to obtain in the future quick and reliable microbiological results in patients with VAP. qPCR can provide reliable quantitative microbiological data, highly specific and with a good sensitivity for common pathogens involved in VAP.

Keywords: pseudomonas aeruginosa; ventilator associated pneumonia; molecular quantification; PICU; VAP 



\section{Introduction}

Ventilator- associated pneumonia (VAP) is the most common nosocomial infection in critically ill patients. Between $10 \%$ and $20 \%$ of the patients receiving more than $48 \mathrm{~h}$ of mechanical ventilation will develop VAP(1).The onset of VAP can be divided into: early which occurs 48 to $96 \mathrm{~h}$ after intubation and late which occurs more than 96h. Early and late VAP differ in their pathogenesis, microorganisms responsible, antibiotic sensitivity, outcome and treatment (2).The actual mortality rate of VAP is still controversial but can exceed $50 \%$ when the initial therapy is not appropriate (3). Diagnosis of VAP is based on clinical, radiological and biological elements(4).

P. aeruginosa is a common nosocomial pathogen and the second agents isolated from patients with Healthcare Associated Pneumonia (HAP), hospitalized patients are not only at risk of dying from severe illness but also from HAP. Isolation of $P$. aeruginosa from respiratory specimens by bacteriological techniques such as culture on various media and biochemical tests is a routine procedure at medical laboratory. However, these procedures are time consuming, and their sensitivities and rapidity are lower than molecular techniques. (5)

Since infection with multi-drug resistant (MDR) isolates of $P$. aeruginosa among patients with HAP is associated with higher mortality rate, early detection of causative agent and administration of appropriate antibiotic is the effective methods to reduce number of deaths(6). Although BAL culture is a good method for diagnosis of VAP, it is invasive and need clinicians but ETA culture is not invasive, cheaper and not in need for clinicians.(7)

Real- time PCR has the upper hand over the conventional culture in diagnosis of Ventilator Associated Pneumonia (VAP) as PCR ability to extract DNA from samples and its ability to obtain quantification of the organisms in $\mathrm{CFU} / \mathrm{ml}$ and converts it into cycle threshold but microbiological methods has limited sensitivity for culture of anaerobic, Real time PCR turned out to be the sensitive and rapid method for the detection and enumeration of infectious agents in VAP (8).

The aim of this study is to compare the performance of the Real-time quantitative molecular -based method versus conventional culture in diagnosis of 
ventilator associated pneumonia caused by pseudomonas aeruginosa in pediatric patients; and Compare benefit of endotracheal aspirate (ETA) versus a bronchoalveolar lavage (BAL) as samples used for microbiological diagnosis of ventilator associated pneumonia (VAP).

\section{Patients and methods}

This prospective study was carried out in the Clinical and Chemical Pathology and Paediatric departments, Faculty of Medicine, Benha University, during the period from January 2018 to June 2019.The study was conducted on 40 pediatric patients aged from one month to 18 years attending paediatric Intensive Care Unit (PICU) and developed VAP during mechanical ventilation.

Approval of the study was obtained from the Ethical Committee of Benha faculty of the medicine. Written consent were taken from patients

\section{Inclusion criteria}

- Paediatric patients older than one month and up to 18 years.

- Paediatric patients on intubation and mechanical ventilation for at least 48 hours if they met the following criteria: a new or progressive infiltrate on chest radiography associated with at least two of the following criteria: body temperature $\quad>38.5^{\circ} \mathrm{C} \quad$ or $<35.0^{\circ} \mathrm{C}$, leucocytic count $\geq 10.000 / \mathrm{mm}^{3}$ or $\leq 4500 / \mathrm{mm}^{3}$ and new onset of purulent tracheal secretions.

\section{Exclusion criteria}

Pneumonia as admission diagnosis or detected within the first 48 hours after hospitalization

\section{Samples:}

Each patient had 2 samples one was bronchoalveolar lavage and the other was endotracheal aspirate:

Endotracheal aspirate (ETA): was obtained by the nurses (a catheter was inserted $30 \mathrm{~cm}$ into the endotracheal tube and the specimen was suctioned under aseptic conditions).(9)

Bronchoalveolar lavage (BAL): a bronchoscope was introduced via an intratraecheal tube in the breathing duct through an extension tube with successive introduction of five aliquots of $20 \mathrm{ml}$ of salted isotonic saline $\left(37^{\circ} \mathrm{c}\right)$ in the lobe that developed infiltration (9).

BAL and ETA were divided into 2 aliquots one for microbiological methods and the 
other was frozen at $-20{ }^{\circ} \mathrm{C}$ for molecular analysis.

All patients were subjected to full history taking, full clinical examination, chest $\mathrm{X}$ ray, semiquantitative culture of $\mathrm{BAL}$ and ETA on different routine bacteriological media for isolation of the causative organisms and molecular quantification for detection and quantification of $P$. aeruginosa in BAL and ETA samples by real -time PCR.

\section{Methods}

\section{Semiquantitative culture of $B A L$ and} ETA on different routine bacteriological media : VAP was established with a positive semiquantitative culture (10).

\section{Identification of the isolates by; gram} stain; colony morphology; biochemical reactions; $P$. aeruginosa; is coagulase negative ,catalase positive, oxidase positive (deep blue violet colour), indole negative, citrate positive. Growth on TSI shows red slope, without gas production and no $\mathrm{H} 2 \mathrm{~S}$ blackening .

3. Automatic identification and confirmation of $\boldsymbol{P}$. aeruginosa by VITEK $2^{\mathrm{TM}}$ - compact 15 (BIOMERIEUX).
4. Molecular Studies by using Real-time PCR for detection of $P$. aeruginosa oprl gene:

- DNA extraction: DNA extraction from BAL and ETA by QIAmp DNA Mini Kit

- DNA amplification by real-time PCR: Enzymatic amplification of the extracted DNA was performed by PCR: using master mix, specific primers and real-time PCR thermal cycler (Applied Biosystem, Step One Plus $^{\text {TM }}$ Real -Time PCR System Thermal Cycling Block model),(made in Singapore), by Primer Design ${ }^{\mathrm{TM}}$ Ltd(German)

\section{Statistical analysis}

The collected data were tabulated and analyzed using SPSS version 16 software (SpssInc, Chicago, ILL Company, USA). Categorical data were presented as number and percentages. Fisher's exact test (FET) and Kappa test were used to analyze them. Quantitative data were tested for normality using Shapiro- Wilks test assuming normality at $\mathrm{P}>0.05$. they were expressed as mean \pm standard deviation if normally distributed or median and range if not. Student " $\mathrm{t}$ " test was used to analyze normally distributed variables among 2 

independent groups. While non parametric variables were analyzed using Man Whitney $\mathrm{U}$ test for 2 independent groups. ROC curves were constructed to assess the performance of PCR ETA and BAL in

\section{Results}

The study was conducted on 40 pediatric with VAP, 24 were males and 16 were females. The mean age in patients' group was

40.7 \pm 31.6 months with male predominance (60\%). Demographic criteria of patients present in table 1 and clinical data presented in table 2

ETA samples and BAL samples were submitted for culture and PCR to detect Pseudomonas aeruginosa isolates. Results showed that in ETA samples $22.5 \%$ of samples yield pseudomonas by culture and $27.5 \%$ by PCR, and in BAL samples, $25 \%$ of samples yield pseudomonas by culture and $27.5 \%$ by PCR.

ROC curve analysis showed that PCR ETA can diagnose Pseudomonas aeruginosa in VAP , with $88.9 \%$ sensitivity, $90.3 \%$ specificity, $72.7 \% \mathrm{PPV}$ and $96.5 \% \mathrm{NPV}$. diagnosis of pseudomonas VAP. The accepted level of significance in this work was stated at $0.05(\mathrm{P}<0.05$ was considered significant).
AUC $(95 \% \mathrm{CI})=0.896(0.76-1.0)$ and ROC curve analysis showed that PCR BAL can diagnose Pseudomonas aeruginosa in VAP, with $100 \%$ sensitivity, $96.7 \%$ specificity, $90.9 \%$ PPV and 100\% NPV. AUC $(95 \% \mathrm{CI})=0.983(0.94-1.0)$. figure 1 and 2 .

There is very good agreement between the results of PCR and that of semiquantitative culture for identification of Pseudomonas aeruginosa in BAL samples (Kappa=0.935).There is good agreement between the results of ETA culture and that of BAL culture for identification of Pseudomonas aeruginosa in VAP patients, (Kappa=0.793). There is an excellent agreement between the results of ETA PCR and that of BAL PCR for identification of Pseudomonas aeruginosa in VAP patients (Карpa=1.0). 

Table (1): Clinical data of the 40 studied VAP patients.

\begin{tabular}{|c|c|c|c|}
\hline Variable & & No. $(\mathrm{N}=40)$ & $\%(100 \%)$ \\
\hline \multirow[t]{2}{*}{ Cause of ICU admission } & Medical & 24 & 60.0 \\
\hline & Surgical & 16 & 40.0 \\
\hline \multirow[t]{2}{*}{ Cause of initiation of $\mathrm{MV}$} & Altered ABG & 12 & 30.0 \\
\hline & Difficult breathing & 28 & 70.0 \\
\hline \multirow[t]{2}{*}{ Duration of ICU admission (days) } & Mean \pm SD & $14.9 \pm 6.3$ & \\
\hline & Median (Range) & $14(1-30)$ & \\
\hline \multirow[t]{2}{*}{ Duration of MV (days) } & Mean \pm SD & $7.3 \pm 2.9$ & \\
\hline & Median (Range) & $7(1-14)$ & \\
\hline \multirow[t]{2}{*}{ Color } & Normal & 30 & 75.5 \\
\hline & Cyanotic & 10 & 25.0 \\
\hline \multirow[t]{2}{*}{ Status } & Sedated & 23 & 57.5 \\
\hline & Irritable & 17 & 42.5 \\
\hline \multirow[t]{2}{*}{ Position } & Semi recumbent & 24 & 60.0 \\
\hline & Supine & 16 & 40.0 \\
\hline \multirow[t]{2}{*}{ Coma } & Yes & 15 & 37.5 \\
\hline & No & 25 & 62.5 \\
\hline \multirow[t]{2}{*}{ Accessory muscles } & Yes & 30 & 75.0 \\
\hline & No & 10 & 25.0 \\
\hline \multirow[t]{5}{*}{ Auscultation } & Fine crepitations & 10 & 25.0 \\
\hline & Coarse crepitations & 6 & 15.0 \\
\hline & Wheezes & 8 & 20.0 \\
\hline & Coarse crepitations + wheezes & 13 & 32.5 \\
\hline & Fine crepitations +wheezes & 3 & 7.5 \\
\hline \multirow[t]{2}{*}{ Associated comorbidities } & Yes & 31 & 77.5 \\
\hline & No & 9 & 22.5 \\
\hline \multirow[t]{2}{*}{ Reintubation } & Yes & 26 & 65.5 \\
\hline & No & 14 & 35.5 \\
\hline \multirow[t]{3}{*}{ Chest X-ray } & Lobar consolidation & 30 & 75.0 \\
\hline & Infiltration & 8 & 20.0 \\
\hline & Airspace opacity & 2 & 5.0 \\
\hline \multirow[t]{2}{*}{ Outcome } & Improved & 30 & 75.0 \\
\hline & Outcome & 10 & 25.0 \\
\hline
\end{tabular}

Table (2):Results of semiquantitative culture and PCR for ETA and BAL samples

\begin{tabular}{llll}
\hline Results of ETA & & No. $(\mathbf{N}=\mathbf{4 0})$ & \% (100\%) \\
\hline \multirow{2}{*}{ Culture } & & & \\
& & 9 & 22.5 \\
PCR of ETA & Non pseudomonas & 31 & 77.5 \\
& Pseudomonas & 11 & 27.5 \\
BAL & Non pseudomonas & 29 & 72.5 \\
Culture & & No. $(\mathbf{N}=\mathbf{4 0})$ & \% $(\mathbf{1 0 0 \%})$ \\
& Pseudomonas & 10 & 25.0 \\
PCR of BAL & Non pseudomonas & 30 & 75.0 \\
& Pseudomonas & 11 & 27.5 \\
\hline
\end{tabular}




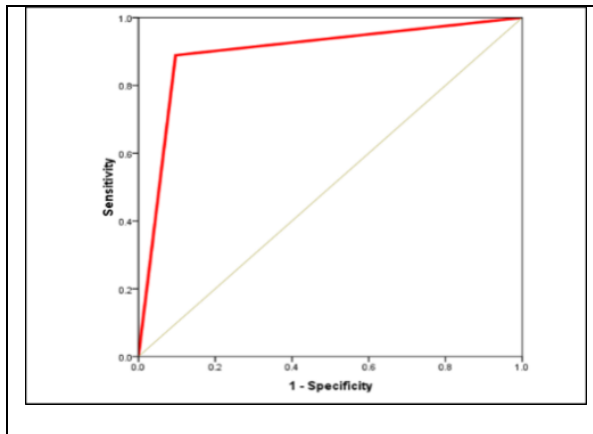

Figure (1): Performance of PCR in detecting Pseudomonas aeruginosa in ETA samples taking semiquantitative culture as a reference method.

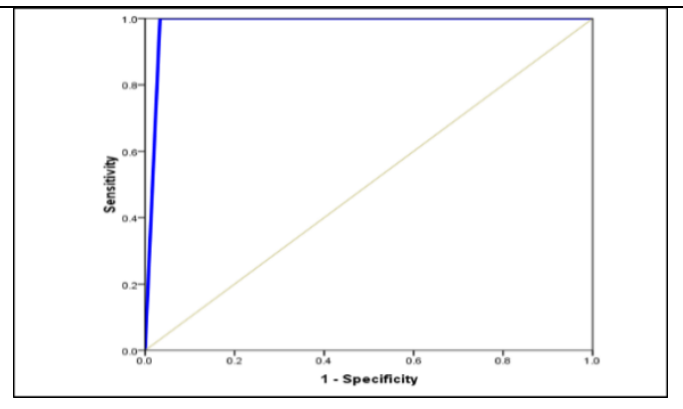

Figure (2): Performance of PCR in detecting Pseudomonas aeruginosa in BAL samples taking semiquantitative culture as a reference method

\section{Discussion}

Ventilator-associated pneumonia (VAP) is a frequent issue in intensive care units (ICU), with a major impact on morbidity, mortality and cost of care. VAP diagnosis remains challenging: traditional culturebased microbiological techniques are still the gold-standard, but are too slow to enable clinicians to improve prognosis with timely antimicrobial therapy adjustment.

The aim of our work is to compare the performance of the real time quantitative molecular based method versus conventional culture in diagnosis of ventilator associated pneumonia caused by $P$. aeruginosa in pediatric patients.

PCR ETA can diagnose Pseudomonas aeruginosa in VAP patients with $88.9 \%$ sensitivity, 90.3\% specificity, $72.7 \%$ PPV and $96.5 \%$ NPV and in BAL samples, with
100\% sensitivity, $\quad 96.7 \%$ specificity, 90.9\% PPV and 100\% NPV. In agreement with our study, some researchers interestingly chose to correlate the cycle threshold $(\mathrm{Ct})$ obtained in real-time quantitative PCR with colony-forming units $\quad(\mathrm{CFU}) / \mathrm{mL}$ obtained from conventional culture. Using this method, the authors report a sensitivity of $89.2 \%$ (range, 83.2-93.6\%) and a specificity of 97.1\% (range, 96.1-97.9\%) using BAL samples, and $71.8 \%$ (range, 61.0-81.0\%) sensitivity and specificity $96.6 \%$ (range, 95.4-97.5\%) using endotracheal aspirates (ETA) (11).

In the present study, there is good agreement between the results of ETA culture and that of BAL culture for identification of $P$. aeruginosa in VAP patients (Kappa=0.793) and this minor 
difference may be due to the high viscosity and heterogeneity of ETA samples which make it difficult to be cultured.

BAL and ETA were compared in 35 mechanically ventilated patients with pneumonia, and reported 93\% agreement between microorganisms cultured from the two types of samples (12).

Real time quantitative diagnosis could offer speed in obtaining results, detection of a very low quantity of target sequences, independency of the viability of target organisms, less effect on results due to the previous use of antibiotics, identification of sequences related to antimicrobial resistance and the possibility of detecting microorganisms difficult to diagnose through conventional methods (14)

In our study, there is a very good agreement between the results of PCR and that of semiquantitative culture for identification of Pseudomonas aeruginosa in BAL samples (Kappa $=0.935$ ). There is a good agreement between the results of ETA culture and that of BAL culture for identification of Pseudomonas aeruginosa in VAP patients (Kappa=0.793) and there is an excellent agreement between the results of ETA PCR and that of BAL PCR for identification of Pseudomonas aeruginosa in VAP patients (Kappa=1.0).
Good concordance between qPCR and conventional culture suggests that $\mathrm{qPCR}$ could provide reliable results to obtain a 'first-line microbiological diagnosis' that would then be made more precise or confirmed by culture results(11)

Detection of bacterial DNA does not always mean infection (15)and in our study, the main challenge for $\mathrm{qPCR}$ is to reduce the number of false-positive results. We obtained one and two false positives for BAL and ETA, respectively. False positive results being one of the main limitations of all qPCR methods currently available, these results showed the importance of quantification that can decrease the number of false positives, as the false positives results in our study are actually true infection as the sensitivity of PCR is higher than the semiquantitative culture.

In the present study, there is negative correlation between cycle threshold and colony forming unit in both BAL and ETA samples. Specific bacterial pathogens related to VAP have been subjected to quantitative studies by molecular techniques. Quantitative PCR results obtained by applying several thresholds in the PCR data were compared with the culture, showing similar results to our 
study and without observed cross reactivity between species (13).

In our study, molecular biology makes it possible to obtain in the future quick and reliable microbiological results in patients with VAP. To be a major clinical advantage, PCR technology has to be available $24 \mathrm{~h}$ per day, the complete automation of the method is required to reduce hands on time and time to result and be applicable in a routine setting (11).

Limitations of our molecular method must be noted; this study was one of the first trying to include a quantitative molecular approach in the microbiological diagnosis of VAP and must be considered as a proof-of-concept study taking in vitro data to the in vivo field. Second, our qPCR designs were restricted to $P$. aeruginosa involved in VAP. Detection and quantification of more pathogens (bacteria, fungi, and viruses) would be required. Finally, the use of our method in the daily practices of a microbiological laboratory is limited today, processing of microbial reference strains in each extraction batch and running of standard curves for quantification

Another major problem with molecular methods is that they do not provide complete susceptibility profiles of bacteria. As bacterial resistance is an important issue in antibiotic treatment, detection of resistance markers is currently being developed for our tool.

Patra et al,(16) reported that VAP constituted $76 \%$ of patients with hospitalacquired pneumonia and represented the most frequent nosocomial infection in intensive care units $(80 \%)$ with an overall mortality of nosocomial pneumonia reaching 60\%; all were secondary to Gram-negative infections with Pseudomonas contributing to $57.1 \%$ of deaths followed by Klebsiella, Escherichia coli and Acinetobacter

In our study the mean duration of ICU admission was $14.9 \pm 6.3$ days and the mean duration of MV was 7.3 \pm 2.9 days. Several studies have showed that prolonged duration of MV and PICU stay were associated with increased VAP risk(17). In the present study there is no significant difference between pseudomonas and non- pseudomonas VAP patients regarding the duration of $\mathrm{MV}$ and length of ICU admission ( $\mathrm{P}>0.05)$. This result was in agreement with others (18).

Micro-aspiration plays a central role in the pathogenesis of nosocomial pneumonia among critically ill patients especially those with nasogastric feeding and on mechanical ventilation (MV). The oropharynx of hospitalized patients 
becomes colonized with aerobic gramnegative bacteria within few days of admission. Therefore, nosocomial pneumonia is caused predominantly by the Gram-negative bacilli (19), within $12 \mathrm{~h}$ of intubation; a biofilm is formed around the endotracheal tube which contains large amounts of bacteria that can be disseminated into the lungs This biofilm may become dislodged during suctioning, or repositioning of the endotracheal tube (20).

Reintubation frequently implicates aspiration of gastric or oropharyngeal contents that are contaminated with colonizing bacteria. many studies highlighted the role of aspiration in the pathogenesis of VAP(21). In our study, reintubation was found not to be a risk factor of VAP $(\mathrm{P}=0.45)$. Similar findings were reported by another study (22). In contrast, it was reported that higher VAP risk with reintubation as impaired mucociliary clearance with mucosal injury and glottic dysfunction associated with prolonged intubation, further aggravates the risk of VAP with re-intubation.

Daily interruption of sedative infusions in critically ill patients receiving mechanical ventilation decreases the duration of mechanical ventilation and reduces the length of ICU stay. Consequently, this practice can be considered worthy for reducing VAP risk and its occurrence (20).

\section{Conclusion}

Molecular biology makes it possible to obtain in the future quick and reliable microbiological results in patients with VAP. qPCR can provide reliable quantitative microbiological data, highly specific and with a good sensitivity for common pathogens involved in VAP.

\section{References}

1. Singh R, Kumar R. A Study of VentilatorAssociated Pneumonia: Incidence, Outcome, Risk Factors And Measures To Be Taken For Prevention. Int $\mathbf{J}$ Med Biomed Stud. 2019;3(10).

2. Elkolaly RM, Bahr HM, El-Shafey BI, Basuoni AS, Elber EH. Incidence of ventilator-associated pneumonia: Egyptian study. Egypt J Bronchol. 2019;13(2):258.

3. Al Ramahi JW, Al-Abdouh A, Hasan N, Haddad G, Al Baba M, Al Aaraj A, et al. Mortality and Length of Stay in Patients with Bloodstream Infections Due to DrugSusceptible Versus Drug-Resistant GramNegative Bacteria. Int $\mathbf{J}$ Infect Dis Ther. 2019;4(3):33.

4. Ceccato A, Panagiotarakou M, Ranzani OT, Martin-Fernandez M, Almansa-Mora R, Gabarrus A, et al. Lymphocytopenia as a predictor of mortality in patients with ICUacquired pneumonia. J Clin Med. 2019;8(6):843.

5. Solanki M, Mehta KD, Sinha M. Pseudomonas aeruginosa in Nosocomial Infection: Burden in Surgical Site of Tertiary Care Unit. Int J Curr Microbiol App Sci. 2018;7(5):2746.

6. Bassetti M, Vena A, Croxatto A, Righi E, Guery B. How to manage Pseudomonas aeruginosa infections. Drugs Context. 2018;7.

7. Patro S, Sarangi G, Das P, Mahapatra A, Mohapatra D, Paty BP, et al. Bacteriological 
profile of ventilator-associated pneumonia in a tertiary care hospital. Indian $\mathbf{J}$ Pathol Microbiol. 2018;61(3):375.

8. Mansour MGE, Albendary S. Multiplex polymerase chain reaction: Could change diagnosis of Ventilator-associated pneumonia in pediatric critical care units to the fast track? Egypt J Med Hum Genet. 2018;19(2):135.

9. Meduri GU, Chastre J. The standardization of bronchoscopic techniques for ventilatorassociated pneumonia. Infect Control Hosp Epidemiol. 1992;13(11):640.

10. Scholte JBJ ,Van Dessel HA. Endotracheal asapirate and bronchoalveolar lavage fluid analysis: interchangeablr diagnostic modalities in suspected ventilator associated pneumonia $.2014: 10.1128 .01494$.

11. Clavel M, Barraud O, Moucadel V, Meynier F, Karam E, Ploy M-C, et al. Molecular quantification of bacteria from respiratory samples in patients with suspected ventilatorassociated pneumonia. Clin Microbiol Infect. 2016;22(9):812.

12. Leone M, Bouadma L, Bouhemad B, Brissaud O, Dauger S, Gibot S, et al. Hospital-acquired pneumonia in ICU. Anaesth Crit Care Pain Med. 2018;37(1):83.

13. Curran T, Coyle P V, McManus TE, Kidney J,Coulter WA. Evaluation of real-time PCR for the detection and quantification of bacteria in chronic obstructive pulmonary disease. FEMS Immunol Med Microbiol. 2007;50(1):112.

14. Murdoch DR, O'Brien KL, Driscoll AJ, Karron RA, Bhat N, Group PMW, et al. Laboratory methods for determining pneumonia etiology in children. Clin Infect Dis. 2012;54(suppl_2):S146.
15.REYGAERT WA. Methicillin-Resistoint Staphylococcus aureus (MRSA): lcentification ond Susceptibility Testing Techniques. Clin Lab Sci. 2009;22(2):120.

16. Patra PK, Jayashree M, Singhi S, Ray P, Saxena AK. Nosocomial pneumonia in a pediatric intensive care unit. Indian Pediatr. 2007;44(7):511.

17. Awasthi S, Tahazzul M, Ambast A, Govil YC, Jain A. Longer duration of mechanical ventilation was found to be associated with ventilator-associated pneumonia in children aged 1 month to 12 years in India. J Clin Epidemiol. 2013;66(1):62.

18. Rello J, Ollendorf DA, Oster G, Vera-Llonch M, Bellm L, Redman R, et al. Epidemiology and outcomes of ventilator-associated pneumonia in a large US database. Chest. 2002;122(6):2115.

19. Cunha BA. Legionnaires' disease: clinical differentiation from typical and other atypical pneumonias. Infect Dis Clin. 2010;24(1):73.

20. Sharma H, Singh D, Pooni P, Mohan U. A study of profile of ventilator-associated pneumonia in children in Punjab. J Trop Pediatr. 2009;55(6):393.

21. Chiru D, Crăciun A, Ţepeneu NF, Şipoş C, Bizerea T, Grecu A, et al. Incidence, Risk Factors, And Nosocomial Germs For Ventilator-Associated Pneumonia In Children. Jurnalul Pediatrului. 2013;16(64).

22. Gautam A, Ganu SS, Tegg OJ, Andresen DN, Wilkins BH, Schell DN. Ventilator-associated pneumonia in a tertiary paediatric intensive care unit: a 1-year prospective observational study. Crit Care Resusc. 2012;14(4):283.

To cite this article: Sahar Fayed, Shahen Dabour, Heba Sallam, Neveen Soliman. Molecular Quantification of Pseudomonas Aeruginosa from Respiratory Samples in Patients with Suspected Ventilator Associated Pneumonia, BMFJ 2021;38(1):210-220. DOI: $10.21608 / \mathrm{bmfj} .2020 .26200 .1232$ 